\title{
Arquitetura Pedagógica Para Aprendizagem Em Makerspaces Educacionais
}

\author{
Karen Selbach Borges ${ }^{1}$, Crediné Silva de Menezes $^{2}$, Léa da Cruz Fagundes ${ }^{2}$ \\ ${ }^{1}$ Instituto Federal de Educação Ciência e Tecnologia do Rio Grande do Sul (IFRS) \\ Câmpus Porto Alegre - RS - Brasil \\ ${ }^{2}$ Universidade Federal do Rio Grande do Sul (UFRGS) \\ Pós-Graduação em Informática na Educação (PGIE) \\ Porto Alegre - RS - Brasil \\ karen.borges@poa.ifrs.edu.br, \{credine, leafagundes\}@gmail.com
}

\begin{abstract}
Resumo: O surgimento dos makerspaces educacionais no Brasil traz consigo o desafio da elaboração de propostas pedagógicas que consigam explorar todo o potencial desses espaços em relação ao desenvolvimento da criatividade e à aprendizagem. Nesse contexto, elaboramos uma proposta de Arquitetura Pedagógica para Aprendizagem em Makerspaces Educacionais (APAME), baseada no uso das tecnologias digitais disponiveis nesses laboratórios e apoiada pela epistemologia de Jean Piaget. A elaboração dessa proposta é fruto de uma pesquisa empírica e espera-se, através da descrição de um exemplo de aplicação da arquitetura pedagógica proposta, mostrar o potencial dos makerspace como espaços de aprendizagem e construção do conhecimento..
\end{abstract}

Palavras-chave: makerspaces educacionais, arquitetura pedagógica

\section{Learning Framework for Educational Makerspace}

\begin{abstract}
The rising of learning makerspace in Brazil brings the challenge of developing educational methodologies that explore the makerspaces' potential as creative and learning spaces. That is why we have developed a Learning Framework for educational makerspace that harness the digital technologies and is supported by Jean Piaget's theories. At the end of this paper, we describe the experience of using the proposed framework and show evidences of learning and knowledge construction.
\end{abstract}

Keywords: learning makerspace, learning framework

\section{Introdução}

O brasileiro é mundialmente reconhecido pela sua criatividade. Entretanto, essa criatividade gera poucos resultados em termos de pesquisa e inovação, as quais são tidas como alguns dos principais elementos para o progresso de um país e para a melhoria da qualidade de vida da sua população (Cornell University; INSEAD; WIPO, 2014). Sabemos das dificuldades políticas e financeiras que nosso país enfrenta para o desenvolvimento da pesquisa e para a transferência do produto dessas pesquisas para a sociedade. Existe, contudo, um problema que é anterior às questões de investimento em 
pesquisa e transferência de tecnologia: a educação. $\mathrm{O}$ modelo educacional vigente, apoiado em uma pedagogia diretiva, representa a "morte da critica, da criatividade, da curiosidade, da inventividade - de tudo aquilo que configura a atividade reflexiva, filosófica ou científica; morte inclusive da pergunta" (Becker, 2012 p.16). Se é a pergunta o elemento que dá início a qualquer projeto de pesquisa e inovação, como esperar que uma educação desse tipo seja capaz de preparar os jovens para serem inventores, cientistas ou inovadores?

Toda pedagogia é legitimada por uma epistemologia, ou seja, em "uma crença sobre a gênese e o desenvolvimento do conhecimento" (Becker, 2012 p. 14). Logo para se chegar à definição de uma pedagogia que favoreça a formação de sujeitos capazes de criar novidades é preciso, primeiro, buscar uma epistemologia que explique como estes sujeitos aprendem e como se tornam capazes de produzir novos conhecimentos e de inventar. A epistemologia genética de Jean Piaget se debruça sobre o desenvolvimento psíquico do sujeito em função da interação com o meio, da maturação e das interações sociais. A interação do sujeito com o meio proporciona experiências que conduzem o sujeito em um processo de equilibração e desenvolvimento de novos esquemas e estruturas cognitivas, que culmina na construção de novos conhecimentos e atos de criatividade. As ideias de Jean Piaget fundamentam o Construtivismo que, com a contribuição de Seymourt Papert, adquiriu um caráter mais pragmático e derivou na pedagogia Construcionista. Essa tem por princípio que

o conhecimento é ativamente construído pela criança em interação com seu mundo, e por isso devemos oferecer oportunidades para que as crianças se envolvam em atividades exploratórias que alimentam o processo construtivo. (tradução nossa) (Ackermann, 2011, p. 1)

O princípio da aprendizagem a partir da experimentação vem tomando força nos dias de hoje através da chamada Educação Mão-Na-Massa (Porvir, 2016). Essa tem se destacado em função do aporte das ferramentas da cultura digital e da aplicação de uma metodologia ativa de aprendizagem que incentiva o protagonismo do aluno, foca o processo ao invés do produto e que vê o erro como oportunidade de crescimento. Várias dessas tecnologias são oriundas de makerspaces. Inicialmente pensados como laboratórios de prototipação, eles têm sido usados como ambientes informais de aprendizagem e, nesse contexto, passaram a ser chamados de Makerspaces Educacionais. Litts (2015) os define como

[...] locais de rica experimentação e inovação, onde os alunos constroem artefatos que fornecem evidências do conteúdo e do processo da aprendizagem, e da sua própria identidade. (tradução nossa) (Litts, 2015, p. 49)

Toda a atividade de aprendizagem em makerspaces faz uso das tecnologias digitais e é essencialmente experimental. O trabalho de Martinez e Stager (2013) apresenta uma proposta para orientar as atividades em makerspaces educacionais denominada TMI (Think, Make, Improve), ou seja, Pensar, Criar e Melhorar. Clap et al. (2016) apresentam uma metodologia de trabalho baseada na inter-relação entre as seguintes habilidades maker: olhar de perto, explorar a complexidade e encontrar oportunidades. Eles propõem rotinas de pensamento (thinking routines) como forma de desenvolver essas habilidades. Já Wardrip e Brahms (2015) propõem desenvolver 
atividades que explorem as práticas de aprendizagem tais como questionar, criar, procurar e compartilhar recursos, desmontar e reutilizar, expressar intenção, desenvolver fluência, e simplificar para complexificar. Essas propostas pedagógicas, documentadas sob o nome de Learning Frameworks, são amparadas no Construcionismo de Papert e foram elaborados levando em consideração o modelo educacional norte-americano e suas normatizações como, por exemplo a Next Generation Science Standards (NGSS, 2013).

Entretanto, no Brasil, por ser uma área ainda pouco explorada pela pesquisa, a aprendizagem em makerspaces educacionais não possui uma proposta pedagógica formalizada, tal como os Learning Frameworks criados pelos pesquisadores norteamericanos. Assim, apresentamos através desse artigo, uma Arquitetura Pedagógica para Aprendizagem em Makerspaces Educacionais (APAME). O objetivo desse trabalho é tornar acessível, a qualquer educador, informações sobre atividades de aprendizagem nesses espaços, fundamentadas por uma epistemologia e apoiadas pelo uso das tecnologias digitais. Na seção 2 introduzimos o conceito de Arquiteturas Pedagógicas. A APAME será detalhada na seção seguinte e um exemplo de aplicação será apresentado na seção 4 . A seção 5 traz as considerações finais e a indicação de trabalhos futuros.

\section{Arquiteturas Pedagógicas}

O modelo educacional alicerçado na transmissão de conhecimento tem se mostrado insuficiente para o desenvolvimento de cidadão autônomos e protagonistas. $\mathrm{Na}$ busca por caminhos mais promissores, tendo em vista os aportes das tecnologias digitais, Carvalho; Nevado; Menezes (2005) introduziram o conceito de Arquiteturas Pedagógicas (AP). As APs são propostas de trabalho estruturantes, elaboradas a partir da vivência do docente, maleáveis e adaptáveis a diferentes enfoques temáticos e diferentes ambientes sócio-educativos, que se apoiam na articulação entre a epistemologia genética de Piaget e a pedagogia da pergunta de Paulo Freire. São princípios de uma AP (Arágon, 2016, p. 262)

a) educar para a busca de soluções de problemas reais; b) educar para transformar informações em conhecimentos; c) educar para a autoria, a expressão e a interlocução; d) educar para a investigação; e f ) educar para a autonomia e a cooperação. (Carvalho, Nevado; Menezes, 2007).

As APs contam com o suporte de tecnologias digitais, as quais, entretanto, "não são definidoras das ações, tampouco consideradas como um simples apoio" (Arágon, 2016, p.263). Elas são um componente importante das APs, servindo tanto como instrumentos cognitivos quanto como ferramenta de mediação pedagógica. As APs pressupõem, não apenas uma adaptação das tecnologias à prática educativa, mas também uma ressignificação de papéis, quando o aluno torna-se protagonista da sua aprendizagem e o professor passa a ser pesquisador e autor de atividades problematizadoras que provoquem, "por um lado, desequilíbrios cognitivos e, por outro, suportes para as reconstruções” (Arágon, 2016, p. 263). 


\section{Arquitetura Pedagógica para Aprendizagem em Makerspaces Educacionais (APAME)}

As APs propõem uma organização de trabalho, cujas atividades envolvem o uso de tecnologias digitais e são amparadas por uma epistemologia de aprendizagem. A figura 1 apresenta, de forma esquemática, como as atividades de aprendizagem em makerspaces podem ser desenvolvidas a partir do desenvolvimento de projetos. Cabe destacar que, no caso da APAME, os projetos têm como objetivo a construção de um artefato físico a partir da articulação de conhecimentos e habilidades nas áreas de design, engenharia e computação.

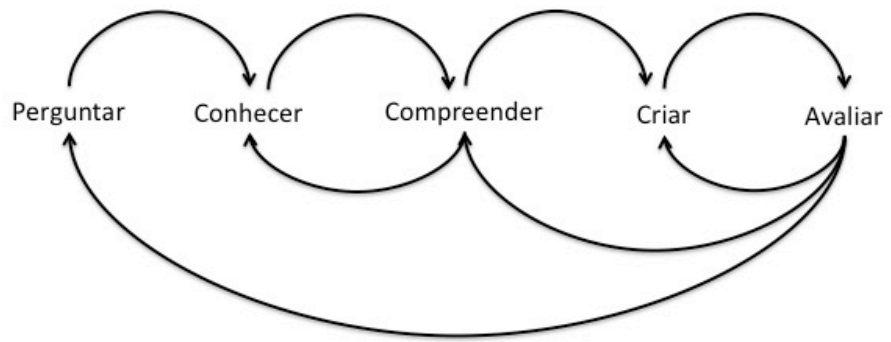

Figura 1 - Fluxo de Atividades

- Perguntar: nessa etapa são identificadas as oportunidades e formuladas as questões que irão orientar o desenvolvimento do projeto. Conforme Fagundes, Sato e Maçada (s.d, p.16) "é fundamental que a questão a ser pesquisada parta da curiosidade, das dúvidas, das indagações do aluno, ou dos alunos, e não imposta pelo professor. Isto porque a motivação é intrínseca; é própria do indivíduo."

- Conhecer: momento em que o sujeito obtém informações mais aprofundadas sobre os elementos (técnicos, humanos e éticos) que compõem o problema. Pode envolver atividades como desmontar um equipamento, pesquisar soluções já existentes, conhecer as tecnologias que poderão ajudar na solução, conhecer as leis relacionadas ao problema, etc.

- Compreender: momento que está intimamente ligado ao conhecer, pois quanto mais se conhece o objeto de pesquisa, melhor se compreende o problema e a solução a qual se deseja chegar. Nesta etapa, alguns conhecimentos tidos como certos podem se mostrar provisório. Nesse caso, é possível que se faça necessário buscar outros conhecimentos que irão ajuda a compreender o problema.

- Criar: corresponde a etapa de fabricação, de correções e ajustes do projeto. $\mathrm{O}$ sujeito torna concreto aquilo que era abstrato. Por causa disso, a avaliação deve ser constante, sendo realizada toda vez que uma parte do projeto toma forma.

- Avaliar: é quando se coloca em uso aquilo que foi criado, sujeitando o objeto a inspeções, testes e comparações. Disso pode resultar a necessidade de correções ou modificações do projeto e até mesmo a necessidade de se compreender melhor os elementos do problema e da solução projetada. Pode ainda, ser o início de um novo ciclo de perguntar, conhecer, compreender, criar e testar. 
A documentação do desenvolvimento dos projetos é parte fundamental dessa AP. Seja na forma de portfólio de projetos ou diário de bordo, o aluno registra a evolução do produto, os processos criativos, de fabricação e de aprendizagem, e os conhecimentos adquiridos ao longo desses processos. Através da documentação o professor pode acompanhar a trajetória de aprendizagem do aluno, encaminhar questionamentos que conduzam o aluno em um processo de reflexão e tomada de consciência, e realizar avaliação contínua sobre as habilidades e competências desenvolvidas pelo aluno. Além disso, a documentação permite o compartilhamento do conhecimento e a divulgação dos resultados obtidos.

\subsection{Suporte Tecnológico}

Essa arquitetura baseia-se no uso de tecnologias, ferramentas e materiais tais como:

- Computadores e/ou dispositivos móveis, através dos quais é possível acessar a internet a fim de realizar busca e compartilhamento de informações; organização e documentação dos projetos; e divulgação dos resultados obtidos.

- Plataformas de desenvolvimento de software: tais como Scratch, Snap!, Aris, S4A, App Inventor, etc. Plataformas mais avançadas podem ser utilizadas dependendo do nível de proficiência em desenvolvimento de software dos envolvidos.

- Plataformas de prototipação eletrônica: Makey-Makey, Arduíno, Lilly Pad, Snap Circuits, entre outros. Essas ferramentas necessitam de baterias, LEDs, resistores, breadboards, jumpers, motores, sensores e atuadores, dependendo do tipo de atividade a ser desenvolvida.

- Máquinas operadas por comandos numéricos $(\mathrm{CNC})$ : impressora 3D, cortadora laser, cortadora de vinil/papel e fresadora. Estas ferramentas trabalham com materiais tais como filamento plástico, MDF, acrílico, couro, vinil, papel, cartolina, EVA, madeira, entre outros.

As máquinas $\mathrm{CNC}$, por possuírem uma interface digital, demandam que os objetos a serem criados sejam previamente projetados no computador, o qual passa a configurar um artefato cognitivo, transcendendo o papel de ferramenta de modelagem. Segundo Lyon (2005) os artefatos cognitivos são dispositivos físicos ou mentais que tem a capacidade de melhorar ou fornecer novas habilidades cognitivas ao sujeito. No caso das ferramentas de modelagem, elas permitem que abstrações internas ao sujeito sejam transformadas em modelos com alto nível de complexidade, descritos na forma de desenhos bi ou tridimensionais, metadados, algoritmos genéticos, redes neurais e gramática de formas (Lyon, 2005). Além de desenvolver a capacidade de abstração, o uso das ferramentas de modelagem demanda que o sujeito desenvolva habilidades típicas do pensamento computacional que, conforme Borges, Menezes e Fagundes (2017), possui estreita proximidade com o pensamento formal.

\subsection{Suporte Teórico}

A APAME está apoiada nas teorias de Jean Piaget sobre aprendizagem, desenvolvimento e construção do conhecimento. Levando em conta que Piaget faz distinção entre aprendizagem lato sensu (aquisição de conteúdos por transmissão ou através da experiência), e stricto sensu (união da aprendizagem lato sensu e dos processos de equilibração) (Piaget, 1974), consideramos que a arquitetura proposta 
contempla ambos os tipos, oferecendo oportunidades de experimentação e momentos de reflexão.

Para Piaget, a experiência física é a base de todo aprendizado, pois propicia as oportunidades de ação, de interação entre o sujeito e os observáveis, proporcionando situações geradoras de desequilíbrios no sistema conceitual do sujeito. A equilibração se dá através de assimilações (incorporação das novas informações ao sistema conceitual pré-existente) e acomodações (formação de novas ligações, expandindo o sistema conceitual). Além disso, quando o sujeito reflete sobre aquilo que observou durante a sua experiência, não apenas para retirar informações a respeito das propriedades dos objetos, mas também a respeito dos resultados de suas ações sobre os objetos, o sujeito precisa raciocinar sobre abstrações, trabalhar com hipóteses e fazer uso de operações lógicas. Dessa forma, o sujeito desenvolve as estruturas cognitivas necessárias para operar de modo formal.

O nível operatório formal representa o mais avançado nível de desenvolvimento cognitivo do sujeito. É caracterizado pela metacognição e pela capacidade do sujeito de operar sobre abstrações e desenvolver raciocínio hipotético-dedutivo. É nesse estágio em que a capacidade criativa dos sujeitos atinge seu ápice, pois

[...] a inteligência consegue, agora, dispensando a interferência do real, dobrar-se sobre si mesma e criar um mundo de significações para além do real, subsumindo o próprio real. Cria um mundo ideal. Esse mundo ideal não se restringe ao passado e ao presente, projeta-se para o futuro. (Becker, 2007 p.45)

\section{Exemplo de Atividade Realizada em um FabLab}

A aplicação da APAME foi realizada no POALab - Fab Lab e contou com a participação de quatro alunos dos níveis técnico e superior tecnológico. A atividade consistia na fabricação de uma máquina capaz de elevar e baixar pequenas peças de madeira. Aos alunos participantes foram disponibilizadas as seguintes ferramentas: cortadora laser, impressora 3D, pistola de cola quente, alicates, estilete, chave de fenda, entre outros instrumentos básicos. Também lhes foi oferecida uma grande variedade de materiais tais como MDF, papelão, isopor, palitos de picolé, fita adesiva, cola quente, grampos, pregos, parafusos, barbante, elástico, fio de nylon, filamento plástico e materiais recicláveis. Conforme o esquema da figura 1, as atividades se desenrolaram da seguinte forma:

- Perguntar: os participantes foram questionados sobre a possibilidade de construir uma máquina capaz de elevar e baixar peças de madeira, utilizando os materiais e ferramentas disponíveis.

- Conhecer: os participantes foram convidados a manipular os materiais e pesquisar sobre suas propriedades. As ferramentas disponíveis no laboratório já eram conhecidas.

- Compreender: os participantes foram instigados a descobrir como os materiais poderiam ser combinados e como as ferramentas poderiam ser utilizadas para produzir o resultado desejado. Como os participantes já tinham conhecimento sobre o uso das ferramentas não foi necessária uma etapa de treinamento. 
- Criar: os participantes trabalharam individualmente para desenvolver uma solução para o problema apresentado. Criaram modelos e protótipos os quais foram documentados utilizando desenhos e fotografias.

- Avaliar: os participantes apresentaram suas criações (figura 2, 3, 4 e 5) e fizeram uma reflexão sobre os pontos fortes e fracos, indicando melhorias possíveis. Também compararam suas soluções com as dos demais colegas.

Do processo de reflexão e avaliação surgiram perguntas como: e se esse protótipo fosse ser transformado em uma máquina real, o que seria necessário mudar? $\mathrm{E}$ se essa máquina além de elevar e baixar peças pudesse também determinar o peso da carga? Como seria possível fazer com o que o sistema de movimento da máquina fosse mecanizado? Essa última questão decorreu do fato de que as soluções apresentadas pelos alunos utilizavam um sistema manual de movimento, como pode ser observado nas figuras 2, 3, 4 e 5 .

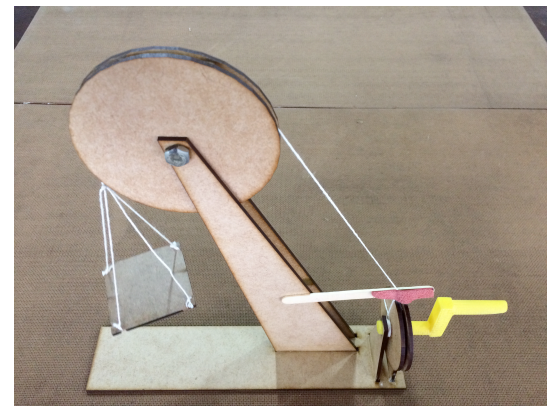

Figura 2 - Criação de João (17)

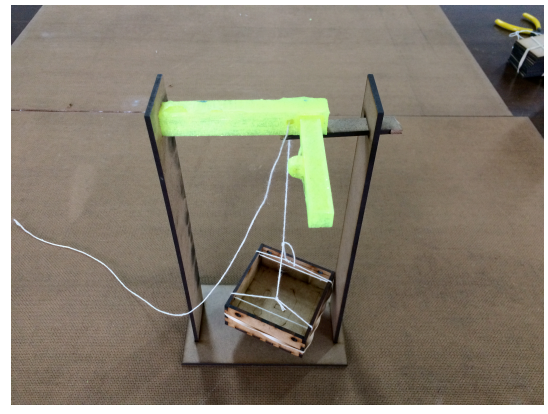

Figura 4 - Criação de Luiz (27)

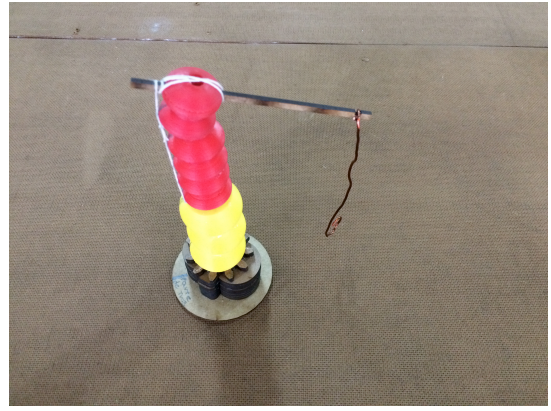

Figura 3 - Criação de Veronica (21)

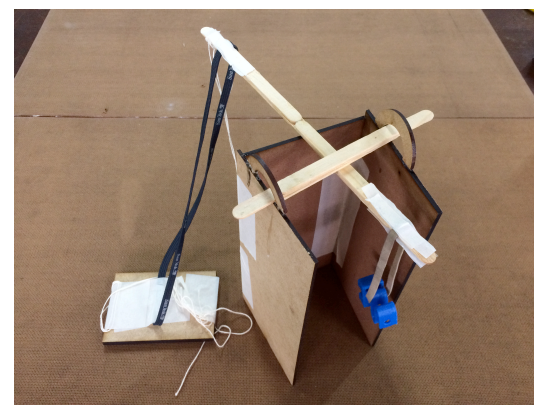

Figura 5 - Criação de Pedro (19)

\subsection{Evidências de Aprendizagem}

A aprendizagem pelo erro é decorrente da reflexão sobre o resultado de uma experiência e está relacionada à aprendizagem lato sensu da qual trata Piaget. É o tipo de aprendizagem mais facilmente identificada e exemplo disso, é o caso de Pedro (19) que modelou uma peça com $20 \mathrm{~cm}$, achando que esse era o tamanho do teclado do computador (referência física que usou para determinar o tamanho da peça que queria cortar em MDF). Entretanto, não mediu o teclado e, depois do MDF cortado, percebeu que a peça ficou pequena. Então resolveu usar régua e conferir a medida do teclado. Em função dessa experiência passou a usar régua e trena para fazer todas as medições necessárias para a modelagem. A aprendizagem decorrente dessa situação relaciona-se com o uso dos instrumentos de medição e a conversão entre medidas (a régua é marcada em centímetros e o software de modelagem utiliza milímetros). 
Já o caso de João (17) está relacionado com a aprendizagem strictu senso, ou seja, é o resultado da reflexão sobre aquilo que foi aprendido com a experiência. Piaget considera isso como parte do processo de abstração reflexiva, a qual constitui a essência dos "atos de criatividade intelectual" (Piaget, 2001, p. 15). João considerava trabalhoso posicionar vários objetos no plano da área de trabalho da ferramenta de modelagem 3D Tinkercad. Tinha que clicar, arrastar e mudar a perspectiva do objeto várias vezes até ter certeza de que o mesmo estava exatamente sobre o plano da área de trabalho (não acima e nem abaixo). Repetia isso para cada um dos objetos. Depois de algumas vezes fazendo isso, desenvolveu uma técnica própria para fazer alinhamento de objetos em 3D. A técnica consiste em criar um cubo, posicioná-lo sobre o plano da área de trabalho e usálo como guia no alinhamento. Por exemplo, para alinhar a base de um cilindro com a área de trabalho, ele insere um cubo no plano da área de trabalho e então utiliza o recurso de alinhamento inferior do Tinkercad sobre o cubo e o cilindro.

Veronica (21), ao ser questionada no início da atividade sobre as variáveis envolvidas no problema cita o peso das peças de madeira que seriam usadas no teste. Ao final, durante a etapa de avaliação, ela comenta "Pois é, aquela hora eu falei peso, né ? Agora eu pensei na altura também", referindo-se a altura da estrutura criada. Mais adiante, durante a conversa, ela acrescenta "podia tentar fazer uma base da estrutura mais forte, para que o peso não acabe levando ela para frente". Isso mostra que, pelo processo de reflexão, Veronica aprendeu que existe uma relação entre o peso da estrutura que irá erguer os objetos e o peso dos objetos em si.

De modo semelhante, Luiz (27), durante o teste da sua estrutura, mostra indícios da aprendizagem sobre a importância da resistência do material a ser utilizado como cabo de sustentação dos pesos. Durante a conversa final foi questionado " $E$ sobre $o$ cabo de sustentação da caixinha, o que tu podes me dizer?" Ele responde "Olha, eu creio que ... eu não sei o quanto de peso aguenta o barbante, mas também ele pode influenciar no quanto mais de peso ela (a estrutura) pode erguer". A resposta, mesmo que ainda sem muita convicção, demonstra que Luiz assimilou a informação sobre a influência da resistência do cabo de sustentação, mas ainda não a acomodou em seu sistema conceitual. Provavelmente, após mais alguns testes, inclusive com materiais diferentes, Luiz conseguiria acomodar a nova informação e, através do processo de reflexão, determinar uma solução melhor.

\section{Considerações Finais}

Os makerspaces constituem importantes ambientes de aprendizagem, uma vez que os projetos desenvolvidos no seu contexto oferecem ricas experiências físicas e lógicomatemáticas, as quais constituem a essência do desenvolvimento humano. Além disso, as interações entre os frequentadores e a aparente desorganização desses espaços, sempre cheios de ferramentas, materiais alternativos para fabricação, protótipos em teste e elementos criativos, tornam os makerspaces um espaço constante de desequilibração. Como diz Menezes et. al. (2006) "ao contrario do que possa parecer, quanto mais complexo o ambiente, no sentido de disponibilizar uma maior interação, maior será a possibilidade de aprendizagem". A atividade descrita na seção anterior nos mostrou que a aprendizagem em makerspaces se dá pela interação do sujeito com o objeto que está sendo criado, mas principalmente através da interação com as tecnologias de fabricação digital. Com o auxílio de simuladores ou ferramentas de modelagem tridimensional, que 
possibilitam a manipulação de perspectivas do objeto, é possível desenvolver elementos do pensamento formal tais como as abstrações e o raciocínio hipotético dedutivo.

Acreditamos que a APAME pode ser utilizada para nortear o desenvolvimento de atividades de aprendizagem em quaisquer áreas do conhecimento. Na Geografia pode-se, por exemplo, desenvolver uma atividade sobre relevo, construindo um modelo tridimensional de determinada região. A reprodução de uma ferramenta medieval pode ser usada como atividade motivadora para uma pesquisa sobre a história daquele período. A criação de novos materiais para impressão 3D é um tema que pode ser trabalhado nas aulas de Química. Deve-se observar, nesses casos, que os projetos são essencialmente interdisciplinares e norteados por uma pedagogia construtivista. Esse casamento entre o currículo escolar (independente do nível educacional) e a Arquitetura Pedagógica para Aprendizagem em Makespaces é um assunto a ser explorado em trabalhos futuros.

\section{Agradecimentos}

Agradecemos ao Instituto Federal de Educação, Ciência e Tecnologia do Rio Grande do Sul, câmpus Porto Alegre, e ao Prof. Dr. André Peres, pela disponibilização do POALab para a realização das atividades descritas.

\section{Referências}

ACKERMANN, E. Piaget's Constructivism, Papert's Constructionism : What's the difference? Disponível em

$<$ http://citeseerx.ist.psu.edu/viewdoc/download?doi=10.1.1.132.4253\&rep=rep1\&typ $\mathrm{e}=\mathrm{pdf}>$. Acesso em outubro de 2017.

ARAGÓN, R. Interação e mediação no contexto das arquiteturas pedagógicas para a aprendizagem em rede. Revista de Educação Pública (UFMT), v25, no. 59, Cuiabá, mai/ago 2016.

BECKER, F. Epistemologia genética e conhecimento matemático. In: BECKER,F. FRANCO, S. R. K. Revisitando Piaget. Porto Alegre: Mediação. 2007, p.22-48

BECKER, F. Educação e Construção do Conhecimento. Porto Alegre: Penso. 2012

BORGES, K.S; MENEZES, C.S; FAGUNDES, L.C. The Use of Computational Thinking in Digital Fabrication projects - a case study from the cognitive persective. In Procedings of Frontiers in Education 2017, p. 93.

CARVALHO, M. J. S.; NEVADO, R. A. de; MENEZES, C. S. de. Arquiteturas Pedagógicas para Educação a Distância: Concepções e Suporte Telemático. In: Anais do XVI SBIE - Simpósio Brasileiro de Informática Na Educação, p. 351360. 2005.

CARVAlHO, M. J. S.; NEVADO, R. A. de; MENEZES, C. S. de. Aprendizagem em Rede na Educação a Distância: estudos e recursos para formação de professores. Porto Alegre: Editora Ricardo Lenz. 2007

CLAP, E. P; et.al. Maker-Centered Learning. Somerset: John Wiley \& Sons, Incorporated. 2016. 
CORNELL UNIVERSITY; INSEAD; WIPO. The Global Innovation Index 2014:

The Human Factor In innovation. Fontainebleau, Ithaca, and Geneva. 2014. Disponível em $<$ http://ideas.repec.org/b/wip/report/2014gii.html $>$. Acesso em setembro de 2017.

FAGUNDES, L. C; SATO, L. S; MAÇADA, D. L. Aprendizes do futuro: as inovações começaram!. MEC - SEAD. Disponível em $<$ http://www.dominiopublico.gov.br/download/texto/me003153.pdf $>$ Acesso em junho de 2017.

LITTS, B. K. Making learning: Makerspaces as learning environments. Universidade de Wisconsin-Madison. 2015. Tese de doutorado. Disponível em $<$ http://informalscience.org/images/research/Litts_2015_Dissertation_Published.pdf $>$ Acesso em junho de 2017.

LYON, E. Autopoiesis and Digital Design Theory: CAD Systems as Cognitive Instruments. International Journal of Architectural Computing, 3(3), 2005. p 317-333. Disponível em <https://doi.org/10.1260/147807705775377366> . Acesso em julho de 2017.

MARTINEZ, S. L.; STAGER, G. Invent to Learn: making, tinkering ans engineering in the classroom. Torrance, CA: Constructing Modern Knowledge Press. 2013.

MENEZES, C. S. de et al. Aplicando Arquiteturas Pedagógicas em Objetos Digitais Interativos. In RENOTE - Revista Novas Tecnologias Na Educação, 4(2), 2006, p. $1-10$

NGSS. Next Generation Science Standard - Executive Summary. 2013. Disponível em

$<$ https://www.nextgenscience.org/sites/default/files/Final\%20Release $\% 20$ NGSS $\% 20$ Front\%20Matter\%20-\%206.17.13\%20Update_0.pdf>. Acesso em junho de 2017.

PIAGET, J; GRÉCCO, P. Aprendizagem e Conhecimento. Rio de Janeiro: Freitas Bastos. 1974

PIAGET, J. Criatividade. In VASCONCELOS, M. S. (org). Criatividade: psicologia, educação e conhecimento do novo. São Paulo: Moderna, 2001. p. 11-20.

PORVIR. Especial Educação Mão Na Massa. 2016. Disponível em $<$ http://porvir.org/especiais/maonamassa/>. Acesso em abril de 2017.

WARDRIP , P . S.; BRAHMS, L. Learning Practices of Making: Developing a Framework for Design. In Proceedings of the 14th International Conference on Interaction Design and Children, 2015, p. 375-378. 